To cite this document: J Anwar-McHenry, RJ Donovan, G Jalleh, A Laws, (2012),"Impact evaluation of the ActBelong-Commit mental health promotion campaign", Journal of Public Mental Health, 11(4), 186 - 194

Permanent link to this document: $h t t p: / / d x . d o i . o r g / 10.1108 / 17465721211289365$

\title{
Impact Evaluation of the Act-Belong-Commit Mental Health Promotion Campaign
}

\author{
Julia Anwar-McHenry*, Robert J. Donovan, Geoffrey Jalleh, Amberlee Laws
}

*corresponding author: julia.anwarmchnry@curtin.edu.au

\begin{abstract}
:
PURPOSE: Act-Belong-Commit is a community-based positive mental health promotion campaign that targets individuals to engage in activities that enhance their mental health while targeting community organisations that provide such activities to promote their activities under the banner of the act-belong-commit message. This paper details key findings from a population impact evaluation of the campaign conducted in 2010.
\end{abstract}

METHODOLOGY: Computer-assisted telephone interviews (CATI) were conducted on a randomly selected adult sample $(N=1,113)$ using quotas to obtain equal representation by age and gender. The questionnaire contained items gauging campaign reach, the impact of the campaign on individual beliefs and behaviours, and perceived societal impact of the campaign on mental illness stigma and openness to mental health issues.

FINDINGS: The campaign reached $75 \%$ of the population. Amongst those exposed to the campaign, $25 \%$ reported changing the way they thought about mental health and mental illness, and $20 \%$ reported some behaviour change. The campaign was perceived to be effective in making people more open about mental health issue (77\% of reached respondents) and reducing stigma surrounding mental illness (68\% of reached respondents).

PRACTICAL IMPLICATIONS: The campaign provides a framework for increasing mental health literacy on a population scale.

ORIGINALITY: With its focus on the positive aspects of mental health, the campaign is unique in promoting behaviours that people can and should do to build and maintain good mental health, while also encouraging community organisations and groups that provide mentally healthy activities to partner with the campaign to make mental health 'everybody's business'. 
To cite this document: J Anwar-McHenry, RJ Donovan, G Jalleh, A Laws, (2012),"Impact evaluation of the ActBelong-Commit mental health promotion campaign", Journal of Public Mental Health, 11(4), 186 - 194

\section{Permanent link to this document: $h$ ttp://dx.doi.org/10.1108/17465721211289365}

\section{Keywords:}

Mental health promotion, campaign evaluation, community-based social marketing

\section{Introduction}

Mental health is an essential resource for productivity, functioning, and wellbeing from individuals to nations (WHO 2005). It has been argued that broad population-based approaches to mental health promotion are needed to progress a reduction in the burden of mental illness which, despite advances in treatment, has not decreased (Kazdin \& Blasé 2011) and is responsible for one third of global disability from ill-health (Jané-Llopis, et al. 2011). Mental health promotion should concern everyone because of the fundamental priority in creating supportive environments to build social, human, and economic capital for the subjective wellbeing of nations (Oishi \& Schimmack 2010; WHO 2005). The call to enhance population wellbeing is based on the argument that a small increase in the population mean of protective factors would have a greater impact on the prevalence of disorder than individual intervention and early prevention with respect to risk factors (Huppert 2009).

A key strategy in the development of community mental health promotion is collaborative community partnerships which are based on existing strengths and resources (Jané-Llopis \& Barry 2005). Furthermore, involving local people in implementation ensures both local commitment and relevance, which are essential for campaign success (Annor \& Allen 2008; Barnett \& Kendall 2011). The need for partnerships with sectors other than health (i.e., sport, recreation, the arts, education, charities, etc.) has also been identified as necessary to more effectively address the social determinants of mental health and wellbeing (Quinn \& Biggs 2010).

Based at Curtin University in Western Australia (WA), Mentally Healthy WA's (MHWA) ActBelong-Commit campaign is a population-based mental health promotion campaign. The campaign combines a community development and social marketing approach to improve community understanding of positive mental health and to involve both health and non-health related service providers in promoting good mental health. The campaign targets individuals to engage in activities that enhance their mental health while encouraging campaign and community partners (e.g., health service providers, local government organisations, and community organisations that provide mentally healthy activities) to promote their activities under the banner of the Act-Belong-Commit 
To cite this document: J Anwar-McHenry, RJ Donovan, G Jalleh, A Laws, (2012),"Impact evaluation of the ActBelong-Commit mental health promotion campaign", Journal of Public Mental Health, 11(4), 186 - 194

\section{Permanent link to this document: http://dx.doi.org/10.1108/17465721211289365}

message (Donovan, et al. 2007; Jalleh, et al. 2007). These collaborating and community partner organisations sign a memorandum of understanding with regard to the promotion of the message and use of the campaign logo. They are also provided with media release templates and other such resources on an ongoing basis for use at their discretion. In addition to any local promotions, publicity, or paid advertising generated by the campaign partners, MHWA used mass media advertising to promote the message with a 30 second television advertisement ${ }^{1}$ broadcast on freeto-air television for a total of 15 weeks over four periods in 2010, a series of three 15 second radio advertisements $^{2}$ on both metropolitan and regional commercial radio stations for five weeks in July and September, and three print advertisements ${ }^{3}$ in five state, regional, and special interest newspapers. Specifically, the campaign messages are that keeping mentally, physically, and socially active (act), participating in group based activities by joining formal and informal community groups (belong), and having meaning and purpose in life by taking up realistic challenges, setting goals, and getting involved in a cause or volunteering (commit) are the foundations of good mental health. There is considerable scientific evidence that these three domains of behaviour contribute to good mental health (see Donovan, et al. 2006b). The execution of this evidence-based campaign was developed primarily from research undertaken by Curtin University into people's perceptions of mental health and the behaviours they believed protected and promoted good mental health (Donovan, et al. 2006a; Donovan, et al. 2003)

The primary connotations of the term "mental health", both by the general public and the health sector itself, are rarely positive, but rather largely generating connotations of "illness" (e.g. schizophrenia, institutions, psychiatrists, etc.) (Donovan, et al. 2003). Hence the campaign organisation was named "Mentally Healthy WA" as formative research revealed this term had positive connotations (e.g. alert, capable, engaged, etc.) (Donovan, et al. 2003).

\footnotetext{
${ }^{1}$ The Act-Belong-Commit cartoon advertisement broadcast in 2010 can be viewed on the Act-BelongCommit website (http://www.actbelongcommit.org.au/resources/adverts/tv-adverts/act-belong-commitcartoon-ad.html). This advertisement was also used in two “Join a Club" television 'doughnut' advertisements in partnership with the WA Department of Sport and Recreation, which can be viewed on Act-Belong-Commit's YouTube channel featuring well-known sports commentators/reporters, Adrian Barich (http://www.youtube.com/watch?v=2fvmz3UgxpY\&feature=plcp) and Michael Thompson (http://www.youtube.com/watch?v=RsTaUyvrlpk\&feature=plcp).

${ }^{2}$ To download or listen to the radio advertisements visit the Act-Belong-Commit website (http://www.actbelongcommit.org.au/resources/adverts/radio-adverts.html).

${ }^{3}$ The print advertisements 'what does it mean to be mentally healthy', 'what makes you happy', and 'get involved' can be downloaded from the Act-Belong-Commit website (http://www.actbelongcommit.org.au/resources/adverts/print-adverts.html)
} 
To cite this document: J Anwar-McHenry, RJ Donovan, G Jalleh, A Laws, (2012),"Impact evaluation of the ActBelong-Commit mental health promotion campaign", Journal of Public Mental Health, 11(4), 186 - 194

\section{Permanent link to this document: http://dx.doi.org/10.1108/17465721211289365}

With MHWA taking on the role of the campaign's research, development, implementation and evaluation hub, partnerships are formed with local and statewide organisations (known as "sites") to deliver the campaign at a local level in a similar way as commercial franchises operate. MHWA provides overall strategic direction, scientific resources, merchandising, mass media advertising, publicity and event sponsorship. In return, and depending on the type of organisation and degree of partnership, organisations promote the message at their events, to their staff, and to their members or clientele.

The population-based campaign was piloted in six communities in regional Western Australia in 2005 before being launched state-wide in 2008 for the three year period 2008 - 2010. The broad target group for mass media advertising is the Western Australian general population. In 2010 there were 24 sites that promoted Act-Belong-Commit across Western Australia (WA). The sites included WA Country Health Service (WACHS) offices, other health services, local governments, recreational, and other not-for-profit organisations, all of whom allocated staff time to manage, deliver and record their sites' activities or in some way promoted the message. In addition, both the hub and individual sites formed collaborative and community partnerships with 36 other organisations, associations, and groups (including recreation, education, sport, cultural, and charity groups) so they could use the educational resources and promote the campaign message in their activities.

This paper reports key findings from a population impact evaluation of the campaign conducted in 2010. Specifically, the evaluation measured population exposure (or reach) of the campaign, the overall impact of the campaign at both an individual level, through the impact on beliefs and behaviours, and at a societal level in reducing perceived stigma and making people more open about mental health issues.

\section{Method}

Computer-assisted telephone interviews (CATI) were conducted statewide with $\mathrm{N}=1,113$ adults: $n=641$ in metropolitan Perth, the state's capital and home to $74 \%$ of the state's population; and $n=$ 472 in country towns (response rate of $48 \%$ ). Country residents were oversampled to provide a sufficient size from which to draw reliable conclusions about country residents. Random selection from the Western Australian White Pages Directories was used to select households for inclusion in the survey. Quotas were used to ensure equal numbers of respondents in two age groups (i.e., 18- 
To cite this document: J Anwar-McHenry, RJ Donovan, G Jalleh, A Laws, (2012),"Impact evaluation of the ActBelong-Commit mental health promotion campaign", Journal of Public Mental Health, 11(4), 186 - 194

\section{Permanent link to this document: http://dx.doi.org/10.1108/17465721211289365}

39 years, $40+$ years), with approximately equal representation of males and females in each age group. The interviews were conducted on weekday evenings between 4:00pm and 8:30pm and on weekends to maximise the availability of household members aged 18 years and over.

Respondents were asked a number of questions to measure exposure to the campaign, Including unaided and prompted awareness of specific campaign elements such as television and radio advertising and the campaign brand/logo (i.e., "Have you seen or heard the following ...."). Respondents reporting any exposure to the campaign were asked whether the campaign had changed the way they think about mental health and mental illness as a result of the messages in the campaign's advertising and publicity ("Have you changed the way you think about mental health and mental illness as a result of becoming aware of the 'Act Belong Commit' messages? If so, "In what way?"), and whether they had changed their behaviour as a result of their exposure to the campaign ("Have you done or tried to do something for your mental health as a result of becoming aware of the Act-Belong-Commit messages?" If so, "What did you do?"). The questionnaire also asked about the campaign's perceived effectiveness with respect to increasing openness towards mental health issues ("Do you think the Act-Belong-Commit campaign will make people more open about mental health issues, less open about mental health issues, or will it make no difference?"), and reducing stigma surrounding mental illness ("Do you think the Act-Belong-Commit campaign will reduce the stigma associated with mental illness, increase this stigma, or will make no difference?").

\section{Results}

\section{Sample Characteristics}

Sample characteristics are shown in Table 1. As per the quota, there were approximately equal numbers of males and females in the sample. Consistent with the census data, the age distribution showed a slightly younger sample in the metro area than in the country, and metro residents were more likely to have a university education compared to country residents.

Occupational status and levels of high school education were similar across both locations and approximately one in five respondents was retired or on a pension. For the analyses detailed below, the data were weighted to reflect the distribution of Western Australia's population of $74 \%$ residing in the metro area and $26 \%$ in the country. Differences between sub-groups on the various measures are reported only where statistically significant. 
To cite this document: J Anwar-McHenry, RJ Donovan, G Jalleh, A Laws, (2012),"Impact evaluation of the ActBelong-Commit mental health promotion campaign", Journal of Public Mental Health, 11(4), 186 - 194

Permanent link to this document: http://dx.doi.org/10.1108/17465721211289365

Table 1: Sample demographics

\begin{tabular}{|c|c|c|}
\hline & Metro & Country \\
\hline & $\mathrm{N}=641$ & $\mathrm{~N}=472$ \\
\hline & $\%$ & $\%$ \\
\hline \multicolumn{3}{|l|}{ Gender: } \\
\hline Males & 49 & 52 \\
\hline Females & 51 & 48 \\
\hline \multicolumn{3}{|l|}{ Age group: } \\
\hline $18-39$ years & 47 & 42 \\
\hline $40+$ years & 53 & 58 \\
\hline \multicolumn{3}{|l|}{ Education level: } \\
\hline Secondary school or less & 17 & 23 \\
\hline Year 12/TEE/TAE & 13 & 15 \\
\hline Some technical or commercial qualifications & 3 & 6 \\
\hline TAFE/Technical qualifications & 22 & 26 \\
\hline Some university & 8 & 10 \\
\hline University degree & 35 & 20 \\
\hline Refused & $<1$ & 0 \\
\hline \multicolumn{3}{|l|}{ Occupation*: } \\
\hline Working full-time & 43 & 49 \\
\hline Working part-time & 20 & 17 \\
\hline Studying full-time & 8 & 3 \\
\hline Studying part-time & 2 & 2 \\
\hline Home duties & 14 & 14 \\
\hline Retired or on a pension & 18 & 20 \\
\hline Unemployed & 1 & 2 \\
\hline
\end{tabular}

* Total may exceed $100 \%$ as multiple responses were permitted.

\section{Campaign reach}

The reach of the campaign was defined as those who had heard of the Act-Belong-Commit campaign, reported seeing the television ad, reported hearing any of the radio ads, or who had seen the logo on any campaign materials. The campaign reach was $75 \%$. As the pilot phase of the campaign was conducted in selected country towns, campaign reach was not unexpectedly slightly higher in the country than in the metro area: $79 \%$ vs. $73 \%\left(\chi^{2}=4.76, p=.03\right)$. This gap has been steadily decreasing since the statewide expansion of the campaign in 2008 (Jalleh, et al. 2009). Campaign reach was significantly higher among females than males $\left(81 \%\right.$ vs. $\left.69 \% ; \chi^{2}=20.35, p=.00\right)$ and among the younger age group (18-39 years) compared with the older age group ( $40+$ years) ( $81 \%$ vs. $70 \% ; \chi^{2}=18.87, p=.00$ ). 
To cite this document: J Anwar-McHenry, RJ Donovan, G Jalleh, A Laws, (2012),"Impact evaluation of the ActBelong-Commit mental health promotion campaign", Journal of Public Mental Health, 11(4), 186 - 194

\section{Permanent link to this document: http://dx.doi.org/10.1108/17465721211289365}

\section{Campaign impact on beliefs and behaviour}

Among those reached by the campaign, $22 \%$ of respondents reported a change in the way they thought about mental health or mental illness (i.e., 17\% of the total sample). This was significantly higher among the younger age group: $28 \%$ vs. $16 \%\left(\chi^{2}=16.86, p=.00\right)$. When asked in what way their beliefs had changed, responses were consistent with the communication objectives of the campaign: increased consciousness about mental health (56\%); various mentions of Act-Belong-Commit activities contributing to good mental health (49\%); and being more understanding of people with mental health problems and the de-stigmatising of people with mental health problems/mental illness (13\%).

With respect to the campaign influencing their behaviour, $20 \%$ of those reached by the campaign (i.e., $15 \%$ of total sample) reported they had done or tried to do something for their mental health. When asked what they had done, the stated behaviours were consistent with the Act-Belong-Commit message: becoming more physically active/increasing exercise (31\%); socialising more/volunteering (29\%); and joining in clubs, groups and community events (21\%).

Those reached by the campaign were also asked whether they had discussed mental health issues with family and friends as a result of the campaign. One in four reached by the campaign (18\% of the total sample) responded that they had, with females more likely to have done so than males: $27 \%$ vs. $21 \%\left(\chi^{2}=3.95, p=.05\right)$.

The above results are summarised in Figure 1 as percentages of the total sample to show population impact.

Figure 1: Overall Population Impact of the Campaign on Beliefs and Behaviour (\% total sample, $n=1113$ )

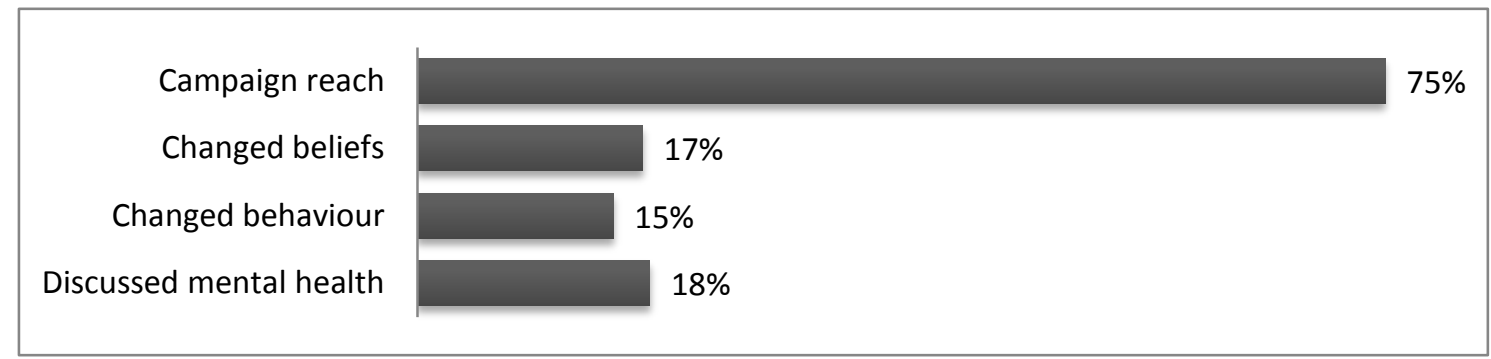


To cite this document: J Anwar-McHenry, RJ Donovan, G Jalleh, A Laws, (2012),"Impact evaluation of the ActBelong-Commit mental health promotion campaign", Journal of Public Mental Health, 11(4), 186 - 194

\section{Permanent link to this document: http://dx.doi.org/10.1108/17465721211289365}

\section{Campaign perceived societal impact re mental health/illness}

To lessen respondent load, two subsamples were chosen at random to answer one or other of the following questions. Approximately half of the campaign reach respondents $(n=380)$ were asked whether they thought the campaign would 'reduce stigma associated with mental illness, increase stigma, or make no difference', while the others $(n=451)$ were asked if they thought the campaign would make people 'more open about mental health issues, less open, or make no difference'. These questions also served to assess whether the campaign had unintended negative effects in these areas.

With respect to openness, just over three in four (77\%) believed the campaign would make people more open about mental health issues (none stated 'less'). A slightly greater proportion of country residents than metro area residents believed this: $84 \%$ vs. $75 \%\left(\chi^{2}=3.90, p=.05\right)$. Approximately two in three (68\%) considered that the campaign would reduce stigma associated with mental illness, with country residents again responding more affirmatively than metro respondents: $77 \%$ vs. $65 \%\left(\chi^{2}=5.33, p=.02\right)$. Importantly, only one percent considered the campaign would increase stigma. These perceived societal impacts are summarised in Figure 2 weighted to the total sample to show population-wide impact.

Figure 2: Overall Campaign Population Perceived Societal Impact re Mental Health/IIIness (\% total sample, $n=1113$ )

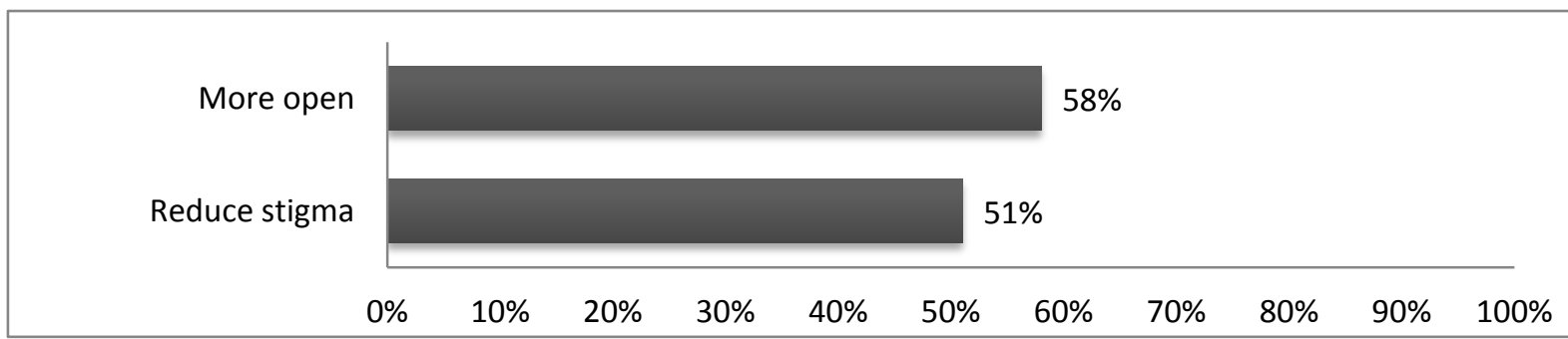

Consistent with these findings, when asked if they perceived that people were 'more willing, less willing, or there was no change in willingness to talk about mental health issues compared to the previous year,' a greater proportion of campaign reach respondents than those not exposed to the campaign reported that people were more willing to talk about mental health issues compared to the previous year: $60 \%$ vs. $45 \%\left(\chi^{2}=19.15, p=.00\right)$. 
To cite this document: J Anwar-McHenry, RJ Donovan, G Jalleh, A Laws, (2012),"Impact evaluation of the ActBelong-Commit mental health promotion campaign", Journal of Public Mental Health, 11(4), 186 - 194

\section{Permanent link to this document: $h$ ttp://dx.doi.org/10.1108/17465721211289365}

\section{Campaign approval}

Finally, when asked whether they 'approved, disapproved or had no opinion either way of campaigns like Act-Belong-Commit that promote what people can do to improve their mental health', almost all respondents (93\%) stated that they approved of such campaigns. Less than two percent of respondents disapproved of such a campaign.

\section{Discussion}

Overall the results reported here generally endorse the Act-Belong-Commit model and messages. There was almost unanimous approval of campaigns such as Act-Belong-Commit, and, despite a limited budget for mass media advertising, the campaign had reached three in four respondents. Given that the media schedule was estimated by the placement company to reach only around 50\% each flight and there were only four three-four week television advertising flights in 2010), these data suggest that other activities such as the WA Health Promotion Foundation (Healthway)'s use of the message in health promotion sponsorship (38 sports and arts events sponsorships in 2010), and delivery of the campaign through sites and partners (a total of 120 in 2010) played an important role in extending the campaign reach.

The campaign message influenced individual beliefs and behaviour with around one fifth of those reached by the campaign reporting a change in beliefs and/or behaviour as a result of exposure to the campaign. The results further show substantial societal impact with over three in four reached respondents perceiving the campaign to make people more open to mental health issues and over two in three perceiving that the campaign would reduce mental illness stigma. This latter result is significant in that the campaign was not directly addressing de-stigmatisation of mental illness and is very different from those that do. Anecdotal feedback suggests that putting mental health in a positive context, as this campaign does, has a significant impact on reducing stigma. Consistent with the above data, campaign reach respondents were also more likely than those not exposed to the campaign to perceive people as more willing to talk about mental health than a year ago. While we cannot assume causality, these data are at least consistent with a campaign exposure effect. 
To cite this document: J Anwar-McHenry, RJ Donovan, G Jalleh, A Laws, (2012),"Impact evaluation of the ActBelong-Commit mental health promotion campaign", Journal of Public Mental Health, 11(4), 186 - 194

\section{Permanent link to this document: http://dx.doi.org/10.1108/17465721211289365}

The impact of the Act-Belong-Commit campaign was substantial in terms of both reach (75\%) and behaviour change (15\%) when compared with the impact of public mental health promotion campaigns reported in the literature. For example, the Californian Friends can be good medicine campaign in the early 1980 s estimated their campaign reach extended to $24 \%$ of the total population (Hunter \& Lloyd-Kolkin 1983) with later campaign evaluation showing a reach of $29 \%$ (Hersey, et al. 1984). Among those reached by the Friends can be good medicine campaign, 65\% (19\% of the total sample) indicated a strong likelihood of engaging in supportive behaviour (Taylor, et al. 1984), although a decay in magnitude of effects was found over time and the evaluation did not account for inherent differences between those reached by the campaign and those who were not (Hersey, et al. 1984). The Australian Together we do better campaign launched in the state of Victoria in 2001 had a prompted recall of $18 \%$ one month after the campaign was launched with $29 \%$ of those who recalled the campaign ( $5 \%$ of the total sample) reporting that they had taken action as a result of the campaign (VicHealth 2001). Apparently no further results have been reported on this campaign. More recently, beyondblue, Australia's National Depression Initiative, achieved a similar campaign reach to Act-Belong-Commit of 76\% in 2007/2008 (an increase from 31\% in 2002). In 2010, beyondblue received around $\$ 29$ million in revenue (beyondblue 2011). The amount spent on media advertising and publicity is unknown, but is presumably substantial. Mentally Healthy WA received $\$ 700,000$ in revenue in 2010 , yet was able to achieve the same level of campaign reach with a media spend of $\$ 300,000$.

There are number of limitations to the evaluation, primarily due to budget limitations on sample sizes. The sample sizes do not allow for statistically reliable analyses of any dose-response differences amongst various sub-groups. The sample size also limits the analyses of differential responses to the campaign by various population subgroups. Such analyses would allow for more strategic planning for various subgroups.

The success of the Act-Belong-Commit campaign arguably stems from a number of factors. First, it was based on extensive formative research with members of the general population as well as stakeholders in mental health/mental illness. The resulting messages were designed to reinforce and increase the salience of factors that most people intuitively believe (and mental health professionals know) are good for their mental health, but which they rarely think about, particularly in a proactive, preventative manner. Second, the campaign targets individuals to change their thinking and do something proactive for their mental health, as well as targeting community organisations that 
To cite this document: J Anwar-McHenry, RJ Donovan, G Jalleh, A Laws, (2012),"Impact evaluation of the ActBelong-Commit mental health promotion campaign", Journal of Public Mental Health, 11(4), 186 - 194

\section{Permanent link to this document: http://dx.doi.org/10.1108/17465721211289365}

provide opportunities for individuals to act on their intentions. Positioning mental health in the same preventative context as physical health not only increases mental health literacy, but also decreases stigma and provides a positive context in which to talk more openly about mental health and mental illness. Finally, the campaign is marked by its use of mass communication strategies enabling broader campaign awareness and population-wide engagement (O'Hara, et al. 2011), along with community development strategies to achieve targeted mental health promotion delivery at a local level.

The success of this campaign model is further evident in the number and types of organisations that have signed on since the statewide expansion began in 2008. The campaign has attracted interest from community groups across a broad variety of institutions (e.g., schools, workplaces, hospitals, etc.) and areas of interest such as sport and recreation, hobbies, the arts, animal protection, walking groups, welfare, and so on, as well as statewide non-health government departments and NGOs. These cover a variety of demographic groups varying in gender, age and socioeconomic status. These partnerships have prompted the development of new initiatives and different modes of partnerships and campaign delivery tailored to specific settings (e.g., seminars for retirees; strategies for assisting in chronic pain control; a self-help guide; activities for children; and so on). Furthermore, despite limited campaign support outside of the state of Western Australia, organisations in all but two states and territories in Australia have signed agreements with MHWA. More recently, a site in the UK has signed an agreement with Mentally Healthy WA to promote ActBelong-Commit to their clients, and the message has been adapted for use with Japanese school children in the aftermath of the 2011 tsunami.

\section{Conclusion}

Calls for population-based approaches to mental health promotion are seen as one solution to progressing a reduction in the burden of mental illness (Huppert 2009; Kazdin \& Blasé 2011). This is partly in response to a recognition that the prevalence of social stigma, misinformation, and overall poor mental health literacy among the general public create barriers and disincentives to not only successful recovery and illness management, but in seeking treatment at all (Smith, et al. 2011; Terry 2009). However, while impacting on all of these factors, the major role for mental health promotion is to strengthen the positive aspects of mental health on a population level. This not only increases resilience to mental health problems, but is a key element for community resilience, individual 
To cite this document: J Anwar-McHenry, RJ Donovan, G Jalleh, A Laws, (2012),"Impact evaluation of the ActBelong-Commit mental health promotion campaign", Journal of Public Mental Health, 11(4), 186 - 194

\section{Permanent link to this document: http://dx.doi.org/10.1108/17465721211289365}

wellbeing, and quality of life via a flourishing, rather than languishing population (Donovan, et al. 2003; Friedli 2009; Friedli, et al. 2007).

The evaluation of mental health promotion is commonly thought to be inherently difficult as a result of the contested nature of mental health as a concept and the multi-dimensional nature of possible outcomes (Friedli \& Parsonage 2007). However, this evaluation demonstrates the effectiveness of the Act-Belong-Commit campaign as a model for mental health promotion on a population-based scale. The campaign is true to its health promoting nature by focusing on strengthening the positive aspects of mental health in a message that is relevant for everyone.

\section{Acknowledgments:}

The Act-Belong-Commit Mentally Healthy WA Campaign is funded largely by Healthway along with substantial support from Lotterywest, the Mental Health Commission and WA Country Health Services.

\section{References}

Annor, S, \& Allen, P. (2008). Why is it difficult to promote public mental health? A study of policy implementation at local level. Journal of Public Mental Health, 7(4), 17-29.

Barnett, L, \& Kendall, E. (2011). Culturally appropriate methods for enhancing the participation of Aboriginal Australians in health-promoting programs. Health Promotion Journal of Australia, 22(27-32).

beyondblue. (2011). Annual Report 2009-10.): beyondblue: the national depression initiative.

Donovan, R J, Henley, N, Jalleh, G, Silburn, S, Zubrick, S, \& Williams, A. (2006a). The impact on mental health in others of those in a position of authority: a perspective of parents, teachers, trainers and supervisors. Australian eJournal for the Advancement of Mental Health, 5(1). Retrieved from www.auseinet.com/journal/vol5iss1/donovan.pdf

Donovan, R J, James, R, \& Jalleh, G. (2007). Community-based social marketing to promote positive mental health: the Act-Belong-Commit campaign in rural Western Australia. In Hastings, $G$ (Ed.), Social Marketing: Why Should the Devil have all the Best Tunes? (pp. 336-343). London: Butterworth Heinemann.

Donovan, R J, James, R, Jalleh, G, \& Sidebottom, C. (2006b). Implementing mental health promotion: the Act-Belong-Commit Mentally Healthy WA campaign in Western Australia. International Journal of Mental Health Promotion, 8(1), 33-42.

Donovan, R J, Watson, N, Henley, N, Williams, A, Silburn, S, Zubrick, S, et al. (2003). Mental Health Promotion Scoping Project.): Report to Healthway. Centre for Behavioural Research in Cancer Control, Curtin University.

Friedli, L. (2009). Mental health, resilience and inequalities.). Copenhagen: World Health Organisation. 
To cite this document: J Anwar-McHenry, RJ Donovan, G Jalleh, A Laws, (2012),"Impact evaluation of the ActBelong-Commit mental health promotion campaign", Journal of Public Mental Health, 11(4), 186 - 194

\section{Permanent link to this document: http://dx.doi.org/10.1108/17465721211289365}

Friedli, L, Oliver, C, Tidyman, M, \& Ward, G. (2007). Mental health improvement: evidence based messages to promote mental wellbeing.). Edinburgh: NHS Health Scotland.

Friedli, L, \& Parsonage, M. (2007). Building an economic case for mental health promotion: part 1. Journal of Public Mental Health, 6(3), 14-27.

Hersey, J C, Kilbanoff, L S, Lam, D J, \& Taylor, R L. (1984). Promoting social support: the impact of California's 'Friends can be good medicine" campaign. Health Education Quarterly, 11(3), 293-311.

Hunter, L, \& Lloyd-Kolkin, D. (1983). Friends can be good medicine: educating the community about social support and health. Paper presented at the American Educational Research Association Annual Conference.

Huppert, F A. (2009). A new approach to reducing disorder and improving well-being. Perspectives on Psychological Science, 4(1), 108-111.

Jalleh, G, Donovan, R J, James, R, \& Ambridge, J. (2007). Process evaluation of the Act-BelongCommit Mentally Health WA campaign: first 12 months data. Health Promotion Journal of Australia, 18(3), 217-220.

Jalleh, G, Donovan, R J, \& Lin, C. (2009). Evaluation of the Act-Belong-Commit Mentally Healthy WA Campaign: 2009 Survey Data.). Perth: Centre for Behavioural Research in Cancer Control, Curtin University.

Jané-Llopis, E, Anderson, P, Stewart-Brown, S, Weare, K, Wahlbeck, K, McDaid, D, et al. (2011). Reducing the silent burden of impaired mental health. Journal of Health Communication, 16(Supplement 2), 59-74.

Jané-Llopis, E, \& Barry, M M. (2005). What makes mental health promotion effective? Promotion and Education, 12(Supplement 2), 47-55.

Kazdin, A E, \& Blasé, S L. (2011). Rebooting psychotherapy research and practice to reduce the burden of mental illness. Perspectives on Psychological Science, 6(1), 21-37.

O'Hara, B J, Bauman, A E, King, E L, \& Phongsavan, P. (2011). Process evaluation of the advertising campaign for the NSW Get Healthy Information and Coaching Service ${ }^{\circledR}$. Health Promotion Journal of Australia, 22, 68-71.

Oishi, S, \& Schimmack, U. (2010). Culture and well-being: a new inquiry into the psychological wealth of nations. Perspectives on Psychological Science, 5(4), 463-471.

Quinn, N, \& Biggs, H. (2010). Creating partnerships to improve community mental health and wellbeing in an area of high deprivation: lessons from a study with high-rise flat residents in east Glasgow. Journal of Public Mental Health, 9(4), 16-21.

Smith, V, Reddy, J, Foster, K, Asbury, E T, \& Brooks, J. (2011). Public perceptions, knowledge and stigma towards people with schizophrenia. Journal of Public Mental Health, 10(1), 45-56.

Taylor, R L, Lam, D J, Roppel, C E, \& Barter, J T. (1984). Friends can be good medicine: an excursion into mental health promotion. Community Mental Health Journal, 20(4), 294-303.

Terry, J. (2009). Mental health first aid - rolling out across the UK. Journal of Public Mental Health, 8(3), 33-37.

VicHealth. (2001). Mental health promotion Wave 1 Survey: Final report.). Carlton: The Wallis Group for VicHealth.

WHO. (2005). Promoting mental health: concepts, emerging evidence, practice.). Geneva: World Health Organization. 